Anthony L. Cunningham, M.B., B.S., M.D.

Westmead Millennium Institute for Medical Research Westmead, NSW, Australia

Thomas C. Heineman, M.D., Ph.D.

GSK Vaccines

King of Prussia, PA

thomas.c.heineman@gsk.com

Since publication of their article, the authors report no further potential conflict of interest.

1. Leroux-Roels I, Leroux-Roels G, Clement F, et al. A phase 1/2 clinical trial evaluating safety and immunogenicity of a varicella zoster glycoprotein E subunit vaccine candidate in young and older adults. J Infect Dis 2012;206:1280-90.

2. Chlibek R, Bayas JM, Collins H, et al. Safety and immunogenicity of an AS01-adjuvanted varicella-zoster virus subunit candidate vaccine against herpes zoster in adults $>=50$ years of age. J Infect Dis 2013;208:1953-61.

3. Chlibek R, Smetana J, Pauksens K, et al. Safety and immunogenicity of three different formulations of an adjuvanted varicella-zoster virus subunit candidate vaccine in older adults: a phase II, randomized, controlled study. Vaccine 2014;32:174553.

DOI: $10.1056 / N E J M c 1508392$

\title{
Short-Course Antimicrobial Therapy for Intraabdominal Infection
}

TO THE EDITOR: Sawyer and colleagues (May 21 issue $)^{1}$ report the findings of the Study to Optimize Peritoneal Infection Therapy (STOP-IT) trial. A strength of this study was the freedom afforded to clinicians in the selection of antibiotic regimens, as long as the choice met Surgical Infection Society-Infectious Diseases Society of America (SIS-IDSA) guidelines. International guidelines vary with respect to recommended regimens for intraabdominal infection ${ }^{2,3}$; this variation partly reflects differences in patterns of antimicrobial resistance worldwide.

Data on the most commonly used antimicrobial agents and culture isolates in the trial are presented in Table S1 in the Supplementary Appendix (available with the full text of the article at NEJM.org), but it would be of value to understand these in more detail, to help clinicians, in particular those outside North America, translate the findings of the trial into clinical practice.

Given the flexibility afforded with respect to first-line therapy, can the authors provide more details on which specific empirical regimens were used in the study? In particular, it would be informative to know the percentage of culture isolates that were susceptible to the initial antimicrobial regimen and how frequently therapy was switched because of resistance. Given the brief duration of treatment in the experimental group, was a mismatch between the choice of the initial antimicrobial drug and organism susceptibility associated with worse outcomes?

Michael Marks, M.B., B.S.

London School of Hygiene and Tropical Medicine

London, United Kingdom

michael.marks@lshtm.ac.uk

Gabriele Pollara, M.B., B.S., Ph.D.

University College London

London, United Kingdom
No potential conflict of interest relevant to this letter was reported.

1. Sawyer RG, Claridge JA, Nathens AB, et al. Trial of shortcourse antimicrobial therapy for intraabdominal infection. N Engl J Med 2015;372:1996-2005.

2. Sartelli M, Viale P, Catena F, et al. 2013 WSES guidelines for management of intra-abdominal infections. World J Emerg Surg 2013;8:3.

3. Solomkin JS, Mazuski JE, Bradley JS, et al. Diagnosis and management of complicated intra-abdominal infection in adults and children: guidelines by the Surgical Infection Society and the Infectious Diseases Society of America. Clin Infect Dis 2010; 50:133-64.

DOI: 10.1056/NEJMc1508694

TO THE EDITOR: Sawyer et al. find that a short course of antimicrobial therapy $(4 \pm 1$ calendar days) was as efficient as an antibiotic treatment guided by a clinical approach with respect to the occurrence of surgical-site infections, recurrent intraabdominal infections, or death. We would like to focus on some points of concern. As designed in the study, this strategy cannot be extrapolated to patients with an inadequate sourcecontrol procedure. The authors did not report the proportion of included patients with severe sepsis, septic shock, or both; mortality among these patients is close to $25 \% .{ }^{1}$ The mortality in this study (0.8 to $1.2 \%$ ) suggests that only patients with uncomplicated intraabdominal infections were involved. Can the study findings be extrapolated to antifungal therapy in Candida albicans infections $(11.2 \%$ of the isolated pathogens in the control group and $7.0 \%$ of the isolated pathogens in the experimental group in this study), given that in such patients the isolation of candida has been considered to be a risk factor for death ${ }^{2}$ In any case, this study should lead physicians to be more cost-effective in their daily practice. 
Claire Roger, M.D.

Martin Bertrand, M.D.

Laurent Muller, M.D., Ph.D.

Nîmes University Hospital

Nîmes, France

claire.roger@chu-nimes.fr

No potential conflict of interest relevant to this letter was reported.

1. Sotto A, Lefrant JY, Fabbro-Peray P, et al. Evaluation of antimicrobial therapy management of 120 consecutive patients with secondary peritonitis. J Antimicrob Chemother 2002;50:569-76.

2. Montravers $\mathrm{P}$, Dupont $\mathrm{H}$, Gauzit $\mathrm{R}$, et al. Candida as a risk factor for mortality in peritonitis. Crit Care Med 2006;34:646-52.

DOI: 10.1056/NEJMc1508694

THE AUTHORS REPLY: To allow for easier participation across multiple sites, details regarding antimicrobial susceptibility were not recorded, and thus our ability to retrospectively determine the adequacy of empirical therapy is limited. As Marks and Pollara point out, in an attempt to limit inadequate initial therapy, participants in the STOP-IT trial were allowed to use any empirical agent that complied with SIS-IDSA guidelines. ${ }^{1}$ However, although the focus on empirical therapy is understandable, it may not be relevant. Bloos et al. found that for patients requiring surgical source control, the only independent risk factor for death at 28 days was a delay in source control of more than 6 hours. Neither the timing of antimicrobial initiation nor the adequacy of empirical treatment was associated with mortality on multivariable analysis. ${ }^{2}$ Our group at the University of Virginia has found similar results. ${ }^{3}$ Regardless, clinicians outside North America should use local guidelines when considering empirical therapy.

In response to Roger et al.: our study was limited to the duration of antimicrobial therapy after diagnosis and source control. Therefore, not only can our results not be applied to pa- tients with inadequate source control, but they also should not be used as evidence for or against specific programs to monitor for infections. The STOP-IT trial did not limit the severity of illness required for enrollment. Subsequently, the Acute Physiology and Chronic Health Evaluation II scores in this trial reflect wide variability in the presentation of intraabdominal infections. This inclusion makes our results more generalizable but less applicable to specific cases such as severe infections and fungal infections.

There is a tendency in the discussion of infectious disease to forget, or rather downplay, that an intraabdominal infection is a surgical disease. Source control, through percutaneous or surgical intervention, is the primary treatment for these patients. Antimicrobial therapy plays an important, albeit secondary, role. Our trial highlights this point by showing that, in patients with adequate source control, the duration of antimicrobial therapy can be halved without any clinically significant change in outcomes.

Christopher A. Guidry, M.D.

Robert G. Sawyer, M.D.

University of Virginia Health System

Charlottesville, VA

rws2k@virginia.edu

Since publication of their article, the authors report no further potential conflict of interest.

1. Solomkin JS, Mazuski JE, Bradley JS, et al. Diagnosis and management of complicated intra-abdominal infection in adults and children: guidelines by the Surgical Infection Society and the Infectious Diseases Society of America. Surg Infect (Larchmt) 2010;11:79-109.

2. Bloos F, Thomas-Rüddel $D$, Rüddel $H$, et al. Impact of compliance with infection management guidelines on outcome in patients with severe sepsis: a prospective observational multicenter study. Crit Care 2014;18:R42.

3. Davies SW, Efird JT, Guidry CA, et al. Does it Matter if we get it right? Impact of appropriateness of empiric antimicrobial therapy among surgical patients. Shock 2014;42:185-91.

DOI: 10.1056/NEJMc1508694

\section{Causality and Chance in the Development of Cancer}

TO THE EDITOR: Luzzatto and Pandolfi (July 2 issue ${ }^{1}$ highlight the combined role of stem-cell turnover, stochastic mutation, and environmental mutagens in the development of cancer. They note the low prevalence of cancer of the small bowel, despite the size of the organ and rapid epithelial turnover. However, among patients with Crohn's disease, the risk of small-bowel adeno- carcinoma is 20 to 30 times that among patients without Crohn's disease. ${ }^{2}$ This disparity in rates illustrates the power of the inflammatory microenvironment to manipulate an apparently genetically stable system. Intestinal inflammation provides a proliferative drive, provokes intestinal stem-cell turnover, and may induce progenitorcell dedifferentiation and intestinal dysbiosis; 